\title{
Tecnura
}

\section{La industria de sensores en Colombia}

\section{The sensors industry in Colombia}

\author{
Angélica Mercedes Nivia Vargas ${ }^{1}$, Iván Jaramillo Jaramillo²
}

Fecha de recepción: 5 de noviembre de 2017

Fecha de aceptación: 15 de marzo de 2018

Cómo citar: Nivia V., A.M. y Jaramillo J., I. (2018). La industria de sensores en Colombia. Tecnura, 22(57), 44-54. DOI: https://doi.org/10.14483/22487638.13518

\section{Resumen}

Contexto: Colombia es un consumidor masivo de productos electrónicos y, en especial, de elementos primarios como sensores; sin embargo, no posee las herramientas para su producción. Los sensores, al ser los elementos necesarios para la realimentación de un proceso, se vuelven imprescindibles para la instauración de cualquier iniciativa de producción.

Método: En este trabajo se realiza un diagnóstico y perspectiva de la industria de sensores en Colombia, enmarcada en la primera etapa de la tesis de maestría titulada "Estrategia de producción de sensores para el sector industrial colombiano". Para tal fin, se hace un breve análisis de entorno (Román, 2016) y se establece su relación con la fabricación de dispositivos electrónicos en Colombia.

Resultados: Se logra identificar algunos aspectos distintivos de la industria, como los sectores económicos donde se ubican las empresas que utilizan sensores, reconocimiento de las variables más medidas y, particularmente, qué sensores son los más utilizados para medir dichas variables. Además, se determinaron las principales compañías proveedoras de sensores en Colombia.

Conclusiones: A partir de la indagación realizada, se concluyó que los sensores que miden temperatura y presión son aquellos con más demanda en la población de la industria nacional, y que el sector al que pertenecen la mayor cantidad de empresas consumidoras de sensores es la manufacturera.

Palabras clave: industria electrónica, instrumentación, perspectiva, sensor.

\begin{abstract}
Context: Colombia is a massive consumer of electronic products, especially primary elements such as sensors, but it does not have the tools to produce them. The sensors, necessary elements for the realization of a process, become essential for the installation of any production initiative.

Method: This paper makes a diagnosis and perspective of the sensor industry in Colombia, framed in the first stage of the Master's thesis entitled "Sensor production strategy for the Colombian industrial sector." For that purpose, an environment analysis is made (Román, 2016), and a relationship between the environment and the manufacture of electronic devices in Colombia is established.
\end{abstract}

Results: It is possible to identify some distinctive aspects of the industry, such as the economic sectors in which companies most use sensors, the identification of the most measured variables, and

1 Ingeniera en Control, magíster en Ingeniería Electrónica (c). Docente Universidad Distrital Francisco José de Caldas. Bogotá D.C., Colombia. Contacto: amniviav@unal.edu.co

2 Ingeniero eléctrico, magíster en Sistemas y Computación. Docente Universidad Nacional de Colombia. Bogotá D.C., Colombia. Contacto: ijaramilloj@unal.edu.co 
particularly which sensors are the most used to measure these variables. Additionally, it was possible to determine which are the main suppliers of sensors in Colombia.

Conclusions: Based on the investigation, it was concluded that the sensors that measure temperature and pressure have the highest demand in the industrial sector, and that the largest number of sensor consuming companies belong to the manufacturing sector.

Keywords: electronics industry, instrumentation, perspective, sensor.

\section{INTRODUCCIÓN}

La industria y la investigación en sensores e instrumentación tienen una influencia muy fuerte ya que existe una gran cantidad de campos en los cuales se requiere la medición de variables, como la automatización, por lo cual uno de los grandes factores de la productividad empresarial depende de esta industria.

En Colombia se requiere el desarrollo de una infraestructura de conocimiento de los sensores para que, en un futuro cercano, se dé origen a una industria nueva y tener las herramientas necesarias para competir en el mundo. $Y$ para llegar a transformar el entorno industrial, se requiere tener el control de cómo se mide y la confianza de los datos obtenidos. Para conseguir esto, es necesario conocer la particularidad del funcionamiento de los elementos de medición, como los sensores, y así estar en la capacidad de aplicar un desarrollo a las necesidades puntuales de cada industria, y provocar una mejora considerable en los procesos industriales desarrollados.

En Colombia se están produciendo cambios de conceptos simultáneos en numerosos campos del conocimiento, que están generando modelos epistemológicos renovados que permiten comprender diferentes fenómenos, a su vez una nueva evolución de tecnologías que surgen del conocimiento de las posibilidades de aplicación. Los avances en campos como la microelectrónica, las telecomunicaciones, la informática, la biotecnología y la robótica, entre otros, están transformando las estrategias de producción concebidas hasta el momento, generando así, valores agregados en la producción de bienes y servicios (Comisión Distrital de Ciencia, Tecnología e Innovación, 2007).

Por esto, pensar en incursionar en una industria propia, como la de la fabricación de sensores, puede ser más factible de lo pensado. El presente trabajo realiza un análisis del estado actual de la industria de sensores en el país, y proporciona un panorama de la potencialidad de crecimiento de esta industria. Un análisis similar y complementario fue desarrollado por el Centro de Investigación y Desarrollo Tecnológico de la Industria Electro Electrónica y TIC CIDEI, en el cual se presentó una perspectiva tecnológica de la industria electro electrónica de Bogotá y Cundinamarca. Para el presente estudio se utilizó una metodología de trabajo similar, sin embargo, el alcance abarca el panorama nacional del uso y fabricación de sensores.

\section{REVISIÓN HISTÓRICA DE LOS SENSORES}

La palabra sensor viene de la raíz latina sentire, que significa percibir. El sensor es considerado como el dispositivo que responde a un estímulo o ante una determinada entrada, y genera una respuesta procesable; esta es referida como mensurando. La respuesta entregada generalmente es una señal eléctrica.

El sensor empezó con la necesidad de cuantificar las variables y es, quizás, el de temperatura el primero en expandir su uso, su surgimiento se remonta al Renacimiento.

Debido a que el calor es una medida de energía en un cuerpo o material, cuanta más energía, más caliente está. Pero, desafortunadamente, las 
propiedades físicas de masa y dimensión eran difíciles de medir. Muchos de los métodos eran indirectos; es decir que para determinar estas variables era necesario observar el efecto que el calor tenía en un objeto y así se deducía la temperatura de este.

El termómetro, como lo conocemos, fue inventado en 1612 en lo que ahora es Italia, por Santorio Santorii. El sensor de temperatura bimetálico fue inventado en el siglo XIX; sin embargo, la electricidad fue una excitante área de investigación y los científicos pronto descubrieron que los metales variaban su resistencia y su conductividad. De ahí que Thomas Johann Seebeck, en 1821, descubriera que se creaba un voltaje cuando la unión de dos metales distintos era ubicada en una temperatura diferente. Más tarde, Peltier descubrió que el efecto del termopar era reversible y podría ser usado con cierta variación como actuador (OMEGA, 2015).

En 1930 el primer termostato de alta temperatura fue introducido al mercado, no obstante, en 1960 fue desarrollado el primer sensor inteligente por Honeywell. Este nació como una solución al problema de compensación de temperatura para el sistema de aire en los aviones DC9; estaba formado por dos piezorresistores que medían la presión y dos capacitores para crear un desplazamiento de fase. Estos elementos estaban realimentados y conectados a un inversor para crear un oscilador. La frecuencia de salida era proporcional a la constante de tiempo y por ende a la presión (Custodio, Bragos y Pallas, 1999).

Tiempo después, Toyota Research presentó otro sensor de presión, similar al desarrollado por Honeywell. Ambas empresas, sin imaginárselo, estaban empezando una revolución sin precedentes en la tecnología de sensado, que aún continúa.

En los últimos años, los sistemas automatizados que realicen diferentes operaciones han tenido un progreso importante, y es de resaltar que los sensores, además de estar presentes en muchas partes de estos sistemas, desempeñan un papel fundamental en su funcionamiento. El sensor está tomando un lugar más importante en las interacciones diarias que cualquier otro dispositivo. Además, se está convirtiendo en parte integral del crecimiento $y$ desarrollo tecnológico.

Específicamente, cada aplicación demanda de varios requerimientos, dentro de los cuales existe por lo menos un sensor que forma parte del sistema. Sin embargo, independientemente de la aplicación, todos los sensores tienen el mismo objetivo: lograr una lectura precisa y estable del mesurando objetivo. De esta manera, la tecnología de sensores ha tenido un crecimiento significativo en el campo de la física, química y la biología.

Hoy, los sensores están encontrando un rol más prominente, la necesidad de aparatos para hacer la vida mejor, más fácil y segura es demasiado fuerte. Estos se utilizan en aplicaciones como: monitoreo ambiental, diagnóstico y atención médica, industria manufacturera y automotriz, electrodomésticos, defensa y seguridad, algunos juguetes, entre otros.

\section{OFERTA DE SENSÓRICA EN COLOMBIA}

El panorama de los sensores está evolucionando; hoy se habla de sensores inteligentes y toda una tecnología de sensado se está fortaleciendo a través de los desarrollos investigativos, por lo cual la necesidad de inversión tanto en calidad como en el desarrollo de sensores está creciendo. Por esto, el pensamiento de los empresarios a la hora de invertir está cambiando y esto se refleja en los productos que se encuentran en el mercado. El mercado cambiante al que se enfrentan las organizaciones está impulsando el desarrollo de ventajas competitivas con miras a mantenerse estables y sobresalir frente a la competencia (De la Hoz, Carrillo y Gómez, 2013).

La vertiginosa evolución de la electrónica, la informática y las telecomunicaciones, su cada vez más requerida demanda, el cambio constante del entorno nacional y mundial, y el impacto que su aplicación tiene en Colombia y el mundo, requieren de nuevos estudios sobre las tendencias, desde el punto de vista tecnológico y de mercados. A partir de esta necesidad, es posible 
reflexionar respecto a las oportunidades que tiene el país de nivelarse con la globalización y los retos que se deben afrontar para lograr una adecuada competitividad.

El Centro de Investigación y Desarrollo Tecnológico de la Industria Electro Electrónica y TIC ClDEI, en alianza con la Asociación Colombiana de Empresas del Sector Electrónico y TIC (ASESEL) realizaron el estudio "Prospectiva tecnológica de la industria electroelectrónica de Bogotá y Cundinamarca", en el cual se hace una revisión del contexto empresarial y científico mundial, y se integra con el componente tecnológico, científico, académico e institucional colombiano. Se pudo identificar que las principales líneas de investigación y tendencias tecnológicas en cuanto a electro electrónica son: nuevos materiales semiconductores, micro- y nanoelectrónica, sistemas micro- y nanoelectrónicos, magnetoelectrónica, bioelectrónica y electromedicina, e integración electrónica fotónica.

Dentro de la industria electrónica mundial se pueden identificar tres tipos de compañías: empresas guía, fabricantes por contrato y líderes de plataforma. La interacción entre ellas determina la evolución de la industria. Las empresas guía son las dueñas de marcas reconocidas, que venden sus productos o sistemas a los consumidores finales $u$ otros negocios; los fabricantes por contrato hacen productos para las empresas guía, y los líderes de plataforma son empresas exitosas en la implantación de tecnología como hardware o software en los productos de otras compañías; un ejemplo es el fabricante de chips Intel (Zuluaga, 2015). Dentro de las empresas guía se pueden distinguir empresas como: Diebold, Siemenes, Rockwell, Phlios, Omron y Dover.

Para identificar aquellas empresas que lideran el mercado de la sensórica en Colombia, se realizó una indagación a grandes y medianas empresas ubicadas en el sector C, D y E, de la Clasificación Industrial Internacional Uniforme (CIIU) (industrias manufactureras, de suministro de electricidad, gas, vapor y aire acondicionado y de distribución de agua; evacuación y tratamiento de aguas residuales), con el propósito de analizar las variables que más se ocupan en medir a lo largo de los procesos de producción y a su vez las principales proveedores de los sensores que utilizan.

Para este ejercicio se tuvo en cuenta una base de datos de 500 empresas suministradas por la Cámara de Comercio de Bogotá y de 673 por el Ministerio de Industria y Comercio. Dentro de los aspectos a consultar se tienen: el sector de la industria de desempeño, variables que más se cuantifican en los principales procesos de producción, variables medidas por el mayor número de sensores, principales proveedores, servicio de los proveedores, referencias de los sensores más comprados, vida útil y calificación de la calidad de los sensores que la compañía más adquiere. Se preguntó por estos elementos mediante un formulario electrónico.

De las respuestas obtenidas, el $30 \%$ de las empresas participantes se ubican en el sector manufacturero; seguido por un $10 \%$ en el sector de minería, áridos y cemento, y un $8 \%$ en el sector de plásticos y cauchos (figura 1).

Teniendo en cuenta que la confiabilidad de los resultados de una encuesta no depende del tamaño de la población encuestada (Triola, 2013), a continuación se describen los resultados obtenidos; a partir de la respuestas dadas a la pregunta “¿Cuál(es) variable(s) requiere(n) ser medida(s) en los principales procesos de producción que tiene su empresa?". Se puede afirmar que las variables más requeridas para ser medidas en un proceso productivo en la industria colombiana son: 1) temperatura, 2) presión, 3) nivel (figura 2).

Las variables que reportan con más equipos medidores en un proceso productivo son: 1 ) temperatura, 2) presión, 3) nivel (figura 3).

De igual manera, se indagó acerca de las empresas proveedoras de la mayor cantidad de sensores para cada caso; como resultado, Siemens se ubica en primer lugar, seguido por Instrumatic (figura 4). 


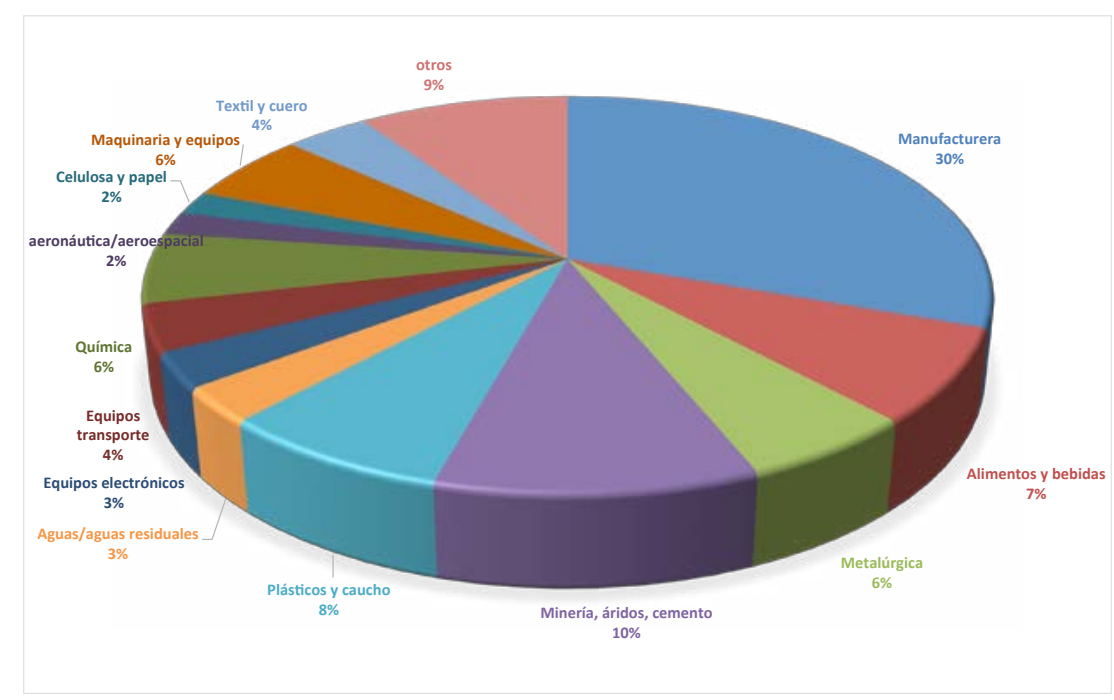

Figura 1. Sectores de la industria de las empresas parte de la muestra.

Fuente: elaboración propia.

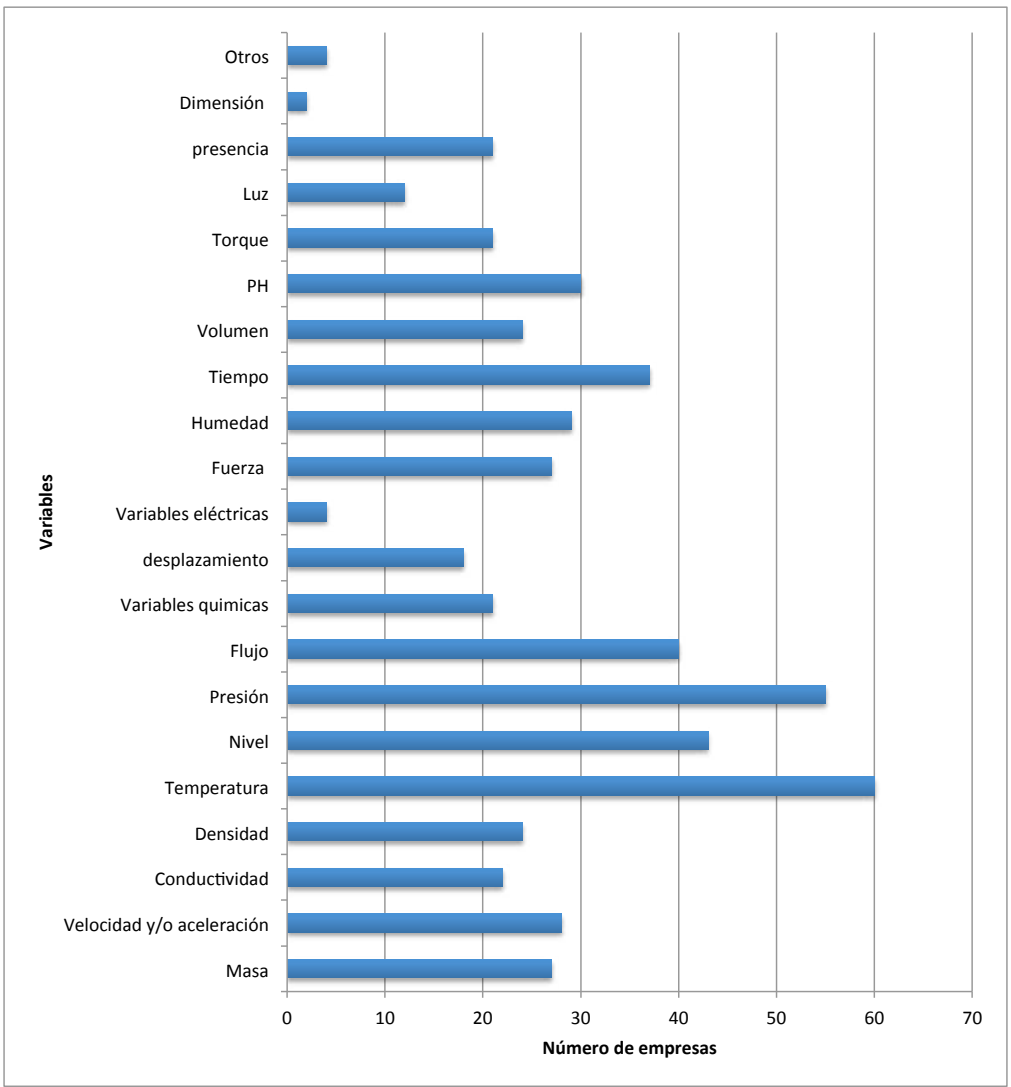

Figura 2. Variables más medidas en un proceso productivo

Fuente: elaboración propia. 


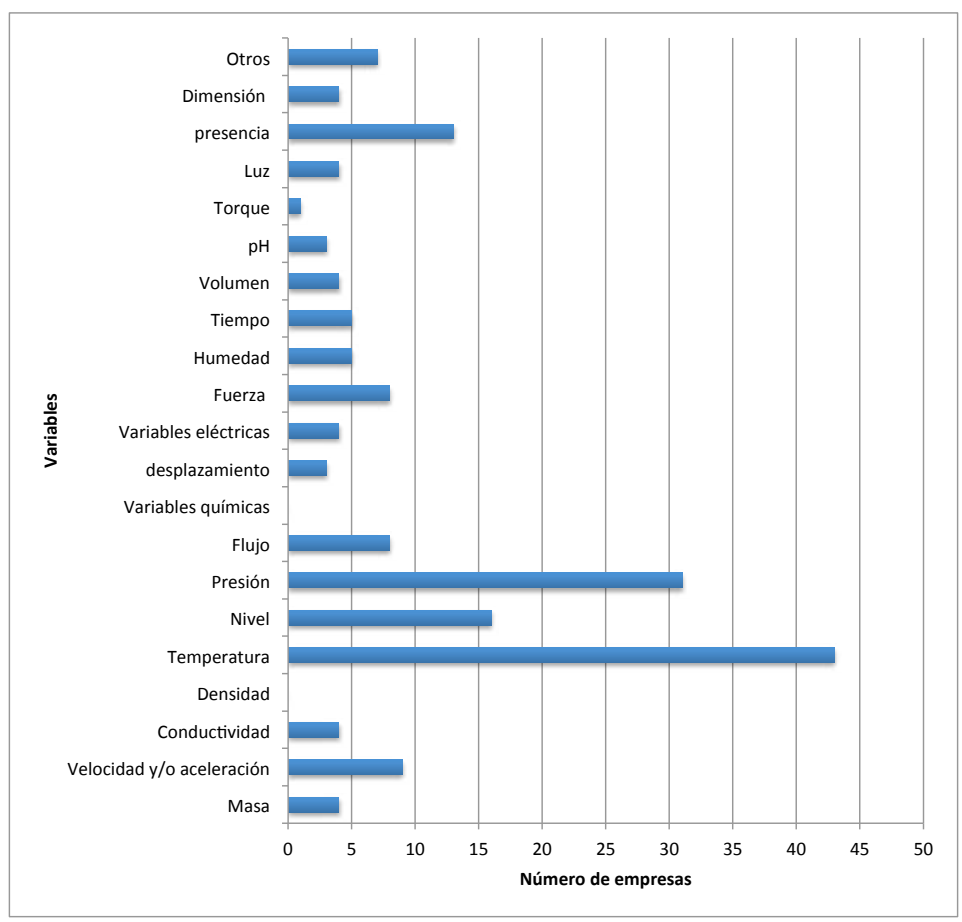

Figura 3. Número de respuestas dadas por empresas del mayor número de sensores por variable que la empresa compra Fuente: elaboración propia.

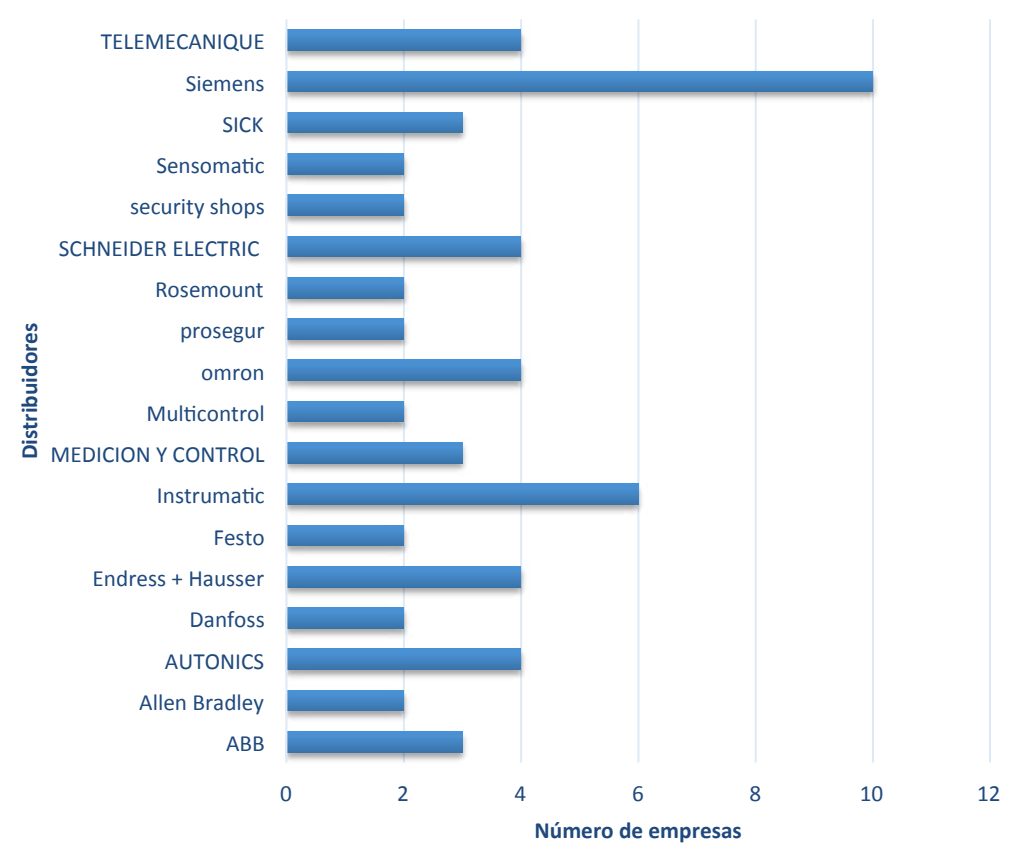

Figura 4. Número de respuestas dadas por empresas de sus distribuidores de sensores más frecuentes

Fuente: elaboración propia. 
Adicionalmente al preguntar a las empresas vendedoras de sensores, indican que los sensores de temperatura son los más vendidos, seguido por los sensores de presencia (figura 5).

\section{DESARROLLO TECNOLÓGICO EN LA INDUSTRIA DE SENSORES}

En Colombia, el avance tecnológico está a cargo de la empresa extranjera, la cual tiene gran participación en nuestra industria a través de la manufactura. Debido a la diversidad de líneas y a los mercados estrechos que posee la industria colombiana, avanzar tecnológicamente no resulta ser fácil. Desarrollar o asimilar tecnología con esfuerzo colombiano es el gran reto por mantener, a través del fortalecimiento de las áreas de investigación en el interior de las empresas (Colciencias, 1994).

Es de gran importancia fortalecer la actividad investigadora dentro de las empresas en desarrollo o con prospección innovadora, en donde muchas veces se requiere una fuerte inversión en el departamento de electrónica. $Y$ a pesar del nacimiento de departamentos de investigación, es uno de los campos más abandonados por las industrias colombianas, ya que sus impactos resultan ser a mediano y largo plazo.

Adicionalmente, se presenta una debilidad financiera de los centros especializados -órganos de creación, difusión y transferencia de conocimiento y tecnología al sector productivo- los cuales fueron creados desde inicios de los noventa, por lo cual no se ha garantizado su normal operación dadas las incertidumbres de apoyo financiero por parte del Estado; por esto, requiere el desarrollo de una industria que demande y produzca conocimiento (Colciencias, 2005).

Hoy, Colombia se limita a propiciar las exportaciones de productos naturales, una serie de insumos de poco valor agregado y mucha inversión de capital. Los requerimientos de inversión tecnológica que se necesitan en las empresas requieren

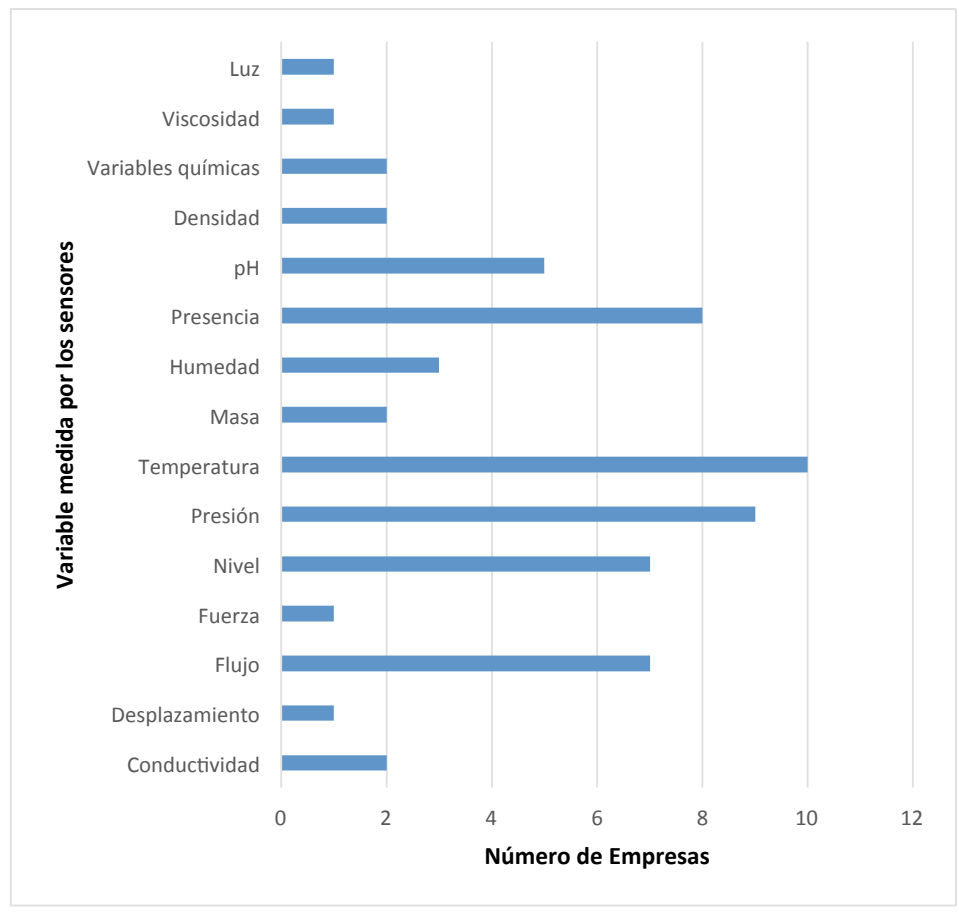

Figura 5. Número de respuestas dadas por empresas vendedoras de los sensores por variable más vendidos

Fuente: elaboración propia. 
de dos aspectos fundamentales: la automatización de procesos y la incorporación de tecnología al producto mismo. Y, en términos generales, resulta más costoso comprar tecnología que desarroIlarla, pero lastimosamente muchas veces se hace necesario hacerlo por falta de disponibilidad de recursos.

Colombia requiere tener empresas con alto valor agregado, de mano de obra y de ingeniería nacional, en la cual se aproveche a los tecnólogos e ingenieros de las ramas especializadas, para que aporten sus conocimientos en la creación de industrias con alto componente tecnológico.

Existe la necesidad de innovación tecnológica, diversificar productos y mantener precios competitivos y no dedicarse solamente al ensamble de productos. Sin embargo, hay una serie de paradigmas acerca de la innovación, que no permiten iniciar una inversión fuerte en tecnología, como: la seguridad que ofrece al comprar tecnología; los países avanzados son los que pueden desarrollar tecnología; el mercado nacional no es suficiente; hay deficiencia de insumos e infraestructura en Colombia; poco personal calificado; entre otros. Sin embargo, puede que sea más rápido el retorno de inversión al comprar tecnología, al no tener el conocimiento profundo del porqué de cada concepto de diseño no es posible evolucionar a largo plazo.

No obstante, como aspecto a resaltar en el transcurso del periodo entre enero y octubre de 2016, las exportaciones no mineroenergéticas representaron el 46,1\% de las exportaciones totales colombianas (Procolombia, 2016), lo que indica que se está fortaleciendo este sector a pesar de los imaginarios con los cuales hoy se trabaja.

Con respecto al área de sensórica, Colombia no tiene una trayectoria significativa en investigación en el área de la electrónica, por lo que no se ha profundizado en gran medida en el campo de los sensores. Las facultades de ingeniería electrónica nacieron a finales de la década de 1950 y mediados de la de 1960, y la actividad de los ingenieros se centraba en la operación de equipos y sistemas, mantenimiento y adopción de algunas tecnologías
(Colciencias, 1993). Y la actualización de los programas universitarios no va a la misma velocidad que la dinámica mundial de la tecnología.

Se requiere fortalecer la estandarización de los diferentes procesos de medición, por lo cual se han incorporado verdaderos sistemas de medición integrales, los cuales requieren el avance constante en procesamiento de datos, tecnología de sensores y diseño de circuitos integrados.

Sin embargo, Colombia está en crecimiento en algunos sectores, como en el de la automatización con cadenas productivas, como metalmecánica y automotriz, fibras, textiles y cementos; algunas empresas que fabrican sistemas de control, robots industriales y sistemas automáticos de almacenamiento, que dan soluciones innovadoras y exportables (Colciencias, 2000).

El Sistema Nacional de Innovación Colombiano, a su vez, ha cambiado en torno al proceso de globalización a partir de la creación de diferentes redes integradas de producción internacional, las cuales se ven identificadas por la presencia creciente de empresas multinacionales que aportan al avance en la organización de la producción y el consumo a nivel mundial; por esto, siempre se busca el funcionamiento mediante economías de escala, ventajas de la especialización y racionalización de costos. Las organizaciones, al atender sus preocupaciones competitivas y por el aprovechamiento óptimo de la tecnología en función de sus objetivos, comprenden que el mejoramiento de sus prácticas, soportadas tecnológicamente, permite aprovechar mejor los recursos y activos de la empresa (Medina, Arévalo y Rico, 2016).

De acuerdo con el Plan estratégico del programa nacional de desarrollo tecnológico, industrial y calidad, publicado por Colciencias (2000), se realizaron investigaciones de la prospectiva en naciones en vía de desarrollo y desarrolladas (figura 6). La informática, tecnologías de la comunicación y electrónica son las actividades prioritarias para los países seleccionados (Estados Unidos, Japón, Reino Unido, Singapur, Corea, Alemania, España e Irlanda) sobre las cuales se realizaron estudios de futuro. 
Actualmente, en Colombia, sectores como el de equipos y tecnologías de comunicación y electrónica ofrecen oportunidades muy probables para el desarrollo en esta área (figura 7) de las tecnologías de la información y la comunicación (TIC), las cuales han permitido el avance favorable en el país. Por su alta correspondencia, se dividen en dos sectores: producción de software, y

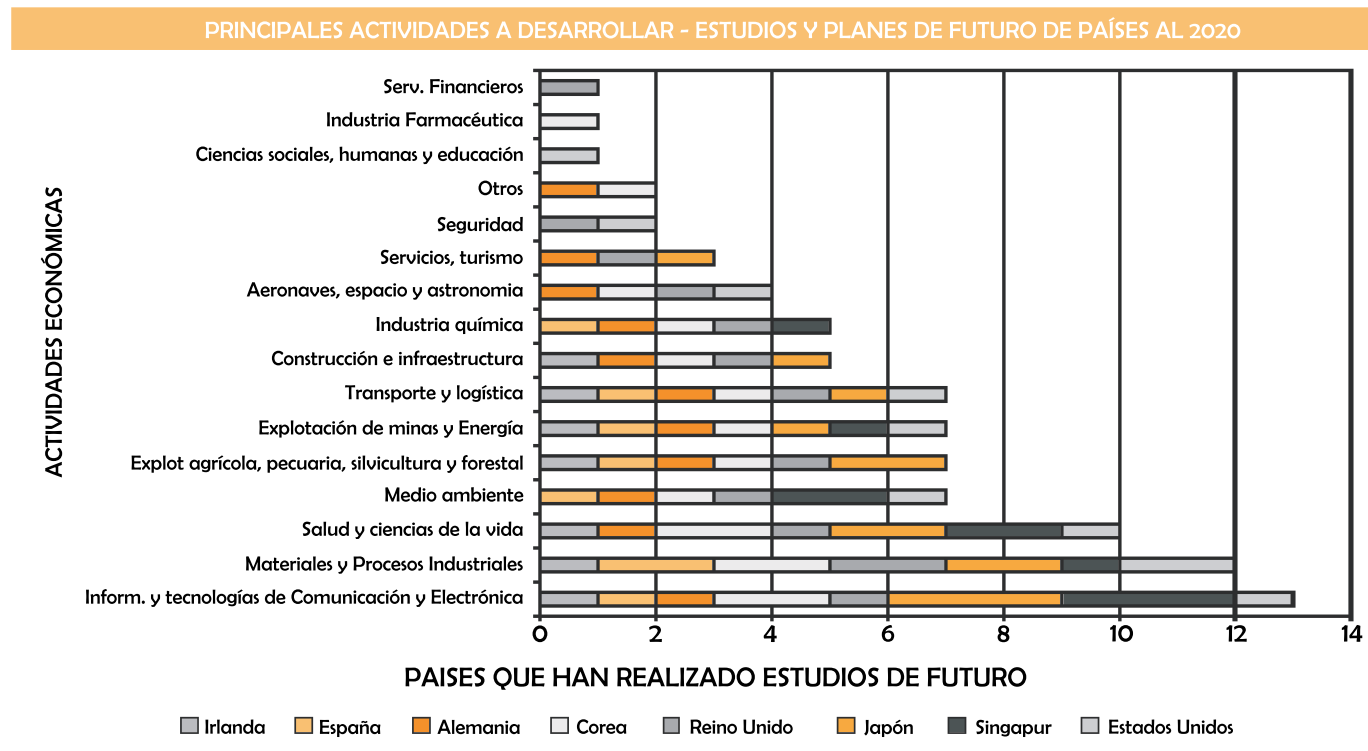

Figura 6. Principales actividades por desarrollar estudios y planes de futuro de países al 2020

Fuente: Colciencias (2000).

\section{ACTUALIDAD Y POTENCIALIDAD PARA EL SECTOR}

\section{EQUIPOS Y TECNOLOGÍAS DE COMUNICACIÓN Y ELECTRÓNICA}

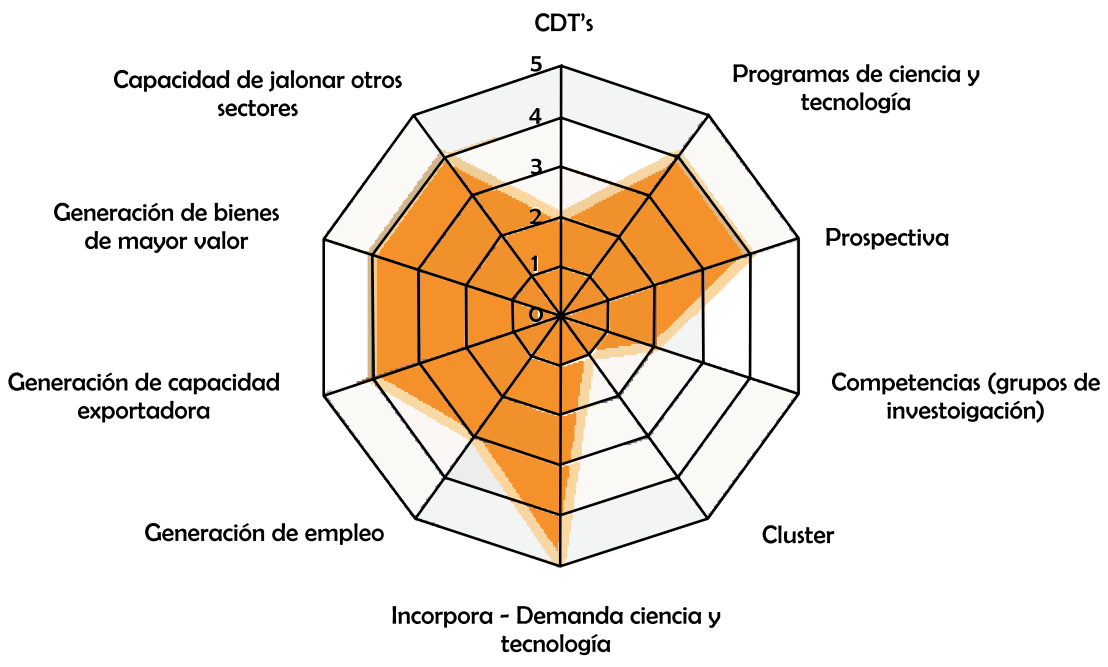

Figura 7. Actualidad y potencialidad para el sector

Fuente: Colciencias (2000). 
producción de equipos y tecnologías de comunicación y electrónica. Para estos dos se concluyen capacidades en el ámbito regional, como Valle, Bogotá y Medellín, en aspectos como educación superior y capacidad empresarial.

Es importante tener en cuenta los aspectos a mejorar, entre ellos, la relación que tienen los grupos de investigación y las universidades en la construcción de asociatividad con el sector productivo, así como con los centros de desarrollo tecnológico y su fuerte capacidad de generar empleo. Este sector tiene la posibilidad de demandar ciencia y tecnología, así como incorporar valor agregado en conocimiento a los bienes y servicios, y a los procesos de otros sectores; en consecuencia, ayuda conjuntamente en el avance y progreso, además es el punto de partida para la investigación en esta área, pudiendo potencializar los procesos productivos y así crear aspectos diferenciadores para una eventual exportación.

\section{PERSPECTIVA Y RETOS DE LA SENSÓRICA}

Sin duda, uno de los elementos más complejos y críticos en las aplicaciones de medición y solución son los sensores. Hay tres parámetros críticos para la utilización del sensor: la sensibilidad, la escala dinámica y la precisión analítica (Acosta, Suárez y Suárez, 2016), por lo cual se requerirán sensores que posean, entre otras características:

- Ancho de banda: valores mínimos hasta varias decenas de $\mathrm{MHz}$.

- Peso: deben ser compactos y ligeros, así como estar hechos de materiales reciclables.

- Precisión: se necesita un valor que garantice una incertidumbre muy pequeña.

- Fiabilidad: garantía de ciclo de vida del producto por lo menos de cinco años, tal como lo reportaron las empresas encuestadas (figura 8).

- Baja tensión: la instrumentación futura será diseñada exclusivamente para ser conectada a sensores de baja tensión, debido a la utilización de los procesadores digitales de señales (DSP) en los instrumentos que requieran baja señal de voltaje.

Por esta razón, la industria de la electrónica, particularmente la sensórica, debe propender por fortalecer su investigación en el área de la medición y de la fabricación de instrumentos, de tal manera que se pueda apropiar la tecnología de fabricación de estos a innovar constantemente

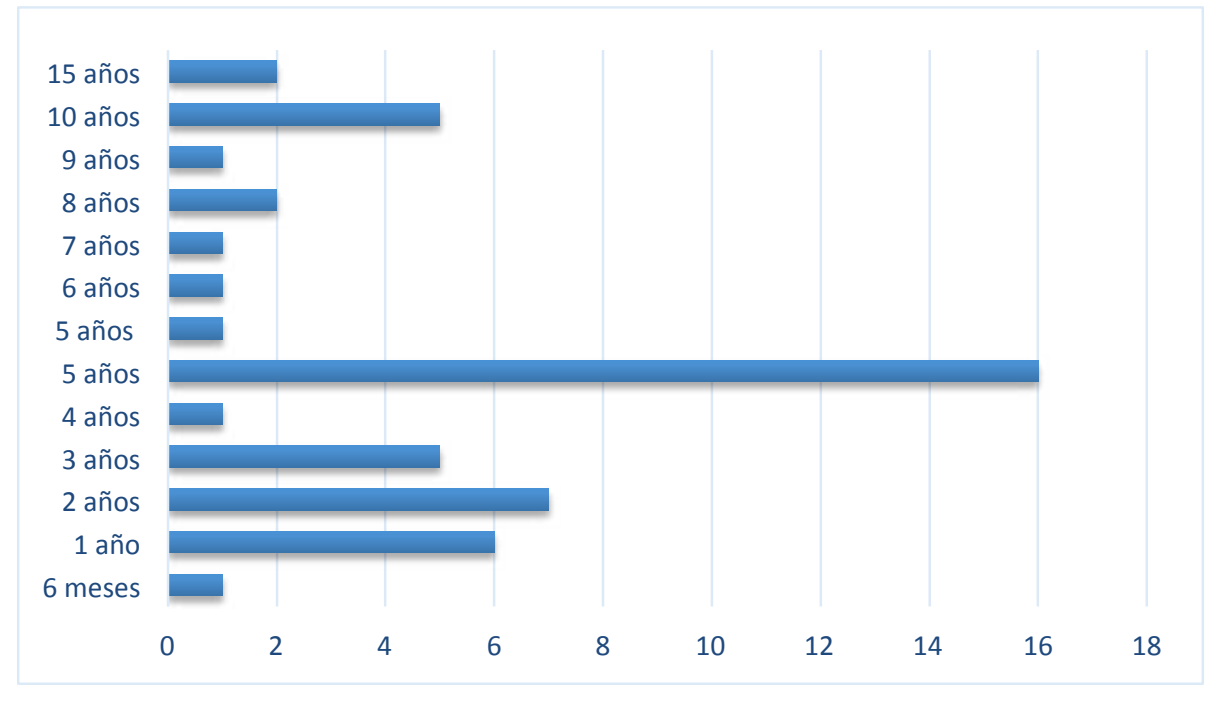

Figura 8. Vida útil esperada de los sensores

Fuente: elaboración propia. 
de acuerdo con las características particulares de nuestro mercado.

\section{CONCLUSIONES}

A partir de las respuestas dadas por los compradores de sensores, se puede afirmar que la variable más presente en los procesos de medición es temperatura, seguido de presión y nivel.

Las universidades colombianas podrían superar los obstáculos para asociarse entre ellas, a través de sus docentes y a su vez con la empresa privada, para llevar a cabo una gran cantidad de proyectos necesarios de manera conjunta universidad/empresa y poder soportar la evolución de la industria y la academia de forma conjunta.

Se requiere que la empresa tenga contacto directo con la dinámica de la investigación, que esté dispuesta a innovar, al riesgo y al emprendimiento a largo plazo.

En un gran número de procesos de producción se debe tener el control de la temperatura, ya que el comportamiento de un sistema puede verse alterado con la variación de esta, razón por la cual pueden ser los sensores más requeridos en la industria.

Colombia tiene un gran potencial para instaurar industrias propias, si reconoce la importancia de invertir en investigación e innovación.

\section{REFERENCIAS}

Acosta, M., Suárez, S. y Suárez, A. (2016). Desarrollo y ensayo de un sensor químico de fibra óptica para la medición de concentraciones de dióxido de carbono. Tecnura, 20(50), 29-42.

Colciencias. (1993). Nuevas tecnologías para la modernización. Bogotá: Tercer Mundo Editores.

Colciencias. (1994). Diez casos exitosos de innovación tecnológica. Bogotá.
Colciencias. (2000). Plan estratégico del programa nacional de desarrollo tecnológico industrial. Bogotá.

Colciencias. (2005). Plan estratégico del programa nacional de desarrollo tecnológico, industrial y calidad. Bogotá.

Comisión Distrital de Ciencia, Tecnología e Innovación (2007). Plan de Ciencia, Tecnología e Innovación Bogotá D.C. 2007 a 2019. Bogotá: Alcaldía Mayor de Bogotá D.C.

Custodio, A., Bragos, R. y Pallas, R. (1999). Sensores Inteligentes: una historia con futuro. Ramas de estudiantes del IEEE, 14, 13 a 18.

De la Hoz, J., Carrillo, E. y Gómez, L. C. (2013). Memorias organizacionales en la era del almacenamiento en la nube. Tecnura, 18(40), 115-126. DOI: https://doi.org/10.14483/udistrital.jour. tecnura.2014.2.a09

Medina, Y., Arévalo, Y. y Rico, D. (2016). Alineación estratégica bajo un enfoque organizacional de gestión tecnológica: ITIL \& ISO 20000. Tecnura, 20, 82-94.

OMEGA (2015). A Brief History of the Temperature Sensor. Recuperado de https://cl.omega.com/technical-learning/pdfs/HistoryofTemperatureSensors_ WhitePaper1.pdf

Procolombia (2016). Análisis de las exportaciones colombianas. Bogotá: Ministerio de Industria y Comercio.

Román, R.E. (2016). Formulación y evaluación de proyectos industriales: creación de nuevas unidades productivas, expansión y/o mejoramiento de empresas en funcionamiento. Bogotá D.C.: Universidad Distrital Francisco José de Caldas.

Triola, M. (2013). Estadística. Naucalpan de Juárez: Pearson.

Zuluaga, D. (2015). Prospectiva tecnológica de la industria electroelectrónica de Bogotá y Cundinamarca. Bogotá D.C.: Fondo de Publicaciones Universidad Sergio Arboleda.

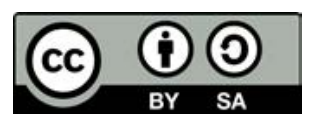

